Volume 3

Issue 4 -- Cardiovascular Aging

$11-11-2016$

\title{
Zero-Fluoroscopy Cavotricuspid Isthmus Ablation Using Carto Mapping System as Sole Guiding Method
}

\author{
Wassim Ballany \\ Indrajit Choudhuri \\ Mohamed Djelmami-Hani \\ Ryan L. Cooley
}

Follow this and additional works at: https://aah.org/jpcrr

Part of the Cardiology Commons, Cardiovascular Diseases Commons, and the Other Analytical, Diagnostic and Therapeutic Techniques and Equipment Commons

\section{Recommended Citation}

Ballany W, Choudhuri I, Djelmami-Hani M, Cooley RL. Zero-fluoroscopy cavotricuspid isthmus ablation using Carto mapping system as sole guiding method. J Patient Cent Res Rev. 2016;3:243-4.

Published quarterly by Midwest-based health system Advocate Aurora Health and indexed in PubMed Central, the Journal of Patient-Centered Research and Reviews (JPCRR) is an open access, peer-reviewed medical journal focused on disseminating scholarly works devoted to improving patient-centered care practices, health outcomes, and the patient experience. 


\section{The Crux of the Heart - the Closest Approach of the Right Atrium to the Left Ventricle}

\author{
Lynn Erickson, David Krum, Hannah Samuel, Anwer \\ Dhala, Jasbir Sra
}

\section{Aurora Research Institute; Aurora Cardiovascular} Services, Aurora Health Care

Background: Accessory pathways, the source of atrioventricular reentry tachycardia, occasionally connect the left ventricle to the right atrium. This is possible because, in some patients, a portion of the right atrium abuts the left ventricle on the posterior wall of the heart, near the mid-coronary sinus. This anatomic region is known as the "crux" of the heart. These accessory pathways can be difficult to ablate because of the unusual and unexpected substrate. While the presence of these accessory pathways is described in the literature, the prevalence of the underlying anatomic substrate is not currently known.

Purpose: To measure the closest approach of the left ventricle to the right atrium by reviewing a consecutive series of standard-of-care computed tomography (CT) scans of the heart taken in patients undergoing interventional cardiac procedures such as catheter ablation of the left atrium.

Methods: Contrast-enhanced cardiac CT exams acquired with electrocardiography-gated retrospective image reconstruction and that had good image quality (i.e. appropriate contrast enhancement of the cardiac structures of interest and low motion artifacts of the acquired images) were reviewed. After scanning the axial images of the relevant anatomy, single or double oblique cuts were made of the three-dimensional data set, and the closest approach of the left ventricle to the right atrium was measured and tabulated.

Results: A total of 47 consecutive CT scans acquired between March 2014 and April 2015 from patients (36 male, 11 female) undergoing catheter ablation for atrial fibrillation were reviewed for this study. The mean distance between the right atrium and left ventricle was $3.6 \pm 1.0 \mathrm{~mm}$. The maximum value was $6.8 \mathrm{~mm}$. For the remaining 46 patients, the right atrium was less than $5.3 \mathrm{~mm}$ from the left ventricle at the point of closest approach. The images showed a single muscular wall between the two chambers at this point, indicating that the right atrium was in contact with the left ventricle.

Conclusion: The right atrium came into direct contact with the left ventricle in all studied patients except one. This has important implications for catheter ablation of certain accessory pathways.

\section{Bias in the Eyes of Resident Physicians}

Abel H. Irena, Kern A. Reid, Richard Battiola, Anthony Cáceres

Department of Internal Medicine, Aurora Sinai Medical Center; Department of Internal Medicine, Aurora UW Medical Group

Background: The utilization of patient characteristics can allow health care providers to arrive at diagnosis or decide on treatment options; however, the subjective nature of patient characterization can negatively affect patient care. A 2003 Institute of Medicine report, called Unequal Treatment, recognized that bias or stereotyping may affect provider-patient communication or the care offered.

Purpose: To investigate residents' recognition of bias in an inpatient care setting.

Methods: In order to explore the topic of bias among providers, we elected to indirectly assess its recognition among providers by asking their opinion in an anonymous manner about their fellow residents. This, we thought, would remove the issue of self-judgment and make it easier for responders to reflect on their observations. We asked residents the following twostep question: "Have you observed a colleague of yours SAY, PORTRAY, or ACT in a biased manner toward a patient while providing inpatient service?" If the answer was yes, we subsequently asked them to elaborate on the bias.

Results: The survey was sent to 39 postgraduate internal medicine residents in their first to third year of training. Half of the responders (20/39) were female. The response rate was $100 \%$, and $46 \%$ (18/39) reported observing their colleague(s) being biased toward patients. Of those who reported bias, $77.8 \%(14 / 18)$ reported one or more examples about the content of the perceived bias. The largest category of these, at $42.8 \%(9 / 21)$, regarded bias toward patients with past or current "drug/substance abuse" or "narcotic seeking" behavior; $14.3 \%$ (3/21) involved patients with repeated admissions or so-called "frequent fliers"; 9.5\% (2/21) related to race/ethnicity; 14.3\% $(3 / 21)$ indicated providers not wanting to care for patients who were perceived to be "difficult." Interestingly, another 9.5\% (2/21) reported witnessing preferential service for "affluent/ VIP" patients. Other examples included bias against obese patients, female patients and general stereotyping with no specifics given.

Conclusion: The majority of resident physicians did recognize bias in their colleagues' approach to patient care. Given the evidence that implicit bias can be recognized and improved upon, this study reinforces the need for implicit bias training/ discussion to be included in residency programs.

\section{Zero-Fluoroscopy Cavotricuspid Isthmus Ablation Using Carto Mapping System as Sole Guiding Method}

Wassim Ballany, Indrajit Choudhuri, Mohamed DjelmamiHani, Ryan L. Cooley

\section{Aurora Cardiovascular Services, Aurora Health Care}

Background: Catheter ablation of the cavotricuspid isthmus (CTI) is traditionally performed using fluoroscopy and electroanatomical mapping systems. Zero-fluoroscopy approaches have recently been studied, mostly using the EnSite ${ }^{\circledR}$ mapping system (St. Jude Medical Inc., St. Paul, MN). We studied the feasibility and efficacy of zero-fluoroscopic mapping and ablation of the CTI using the Carto ${ }^{\circledR} 3$ system (Biosense Webster Inc., South Diamond Bar, CA).

Purpose: To evaluate the efficacy and safety of a zerofluoroscopic approach to CTI ablation. 
Methods: We included 9 patients with typical atrial flutter for whom mapping and ablation of the CTI was done using Carto mapping as the sole guiding modality. Zero fluoroscopy was achieved in all of them. Another group of 9 matched patients whose ablations were done using the traditional method (i.e. fluoroscopy and Carto guidance) were included as a control.

Results: Both groups had similar baseline characteristics. The total fluoroscopy time and radiation dose in the control group were $12.8 \pm 4.8$ minutes, $230.2 \pm 131.9 \mathrm{mGy}$, respectively. The zero-fluoroscopy group were done while wearing no lead aprons. The average total procedure time was $114.8 \pm 16.9$ minutes in the zero-fluoroscopy group, significantly less than that of the control group $(138.6 \pm 24.3$ minutes; $\mathrm{P}=0.0286)$. The total radiofrequency time was similar in both groups $(15.2 \pm$ 7.4 minutes in zero-fluoroscopy group vs $16 \pm 2.9$ minutes in control group; $\mathrm{P}=0.9294)$. Bidirectional block was achieved in all patients of both groups. No complications were encountered in either group.

Conclusion: To our knowledge, this is the first study of a zerofluoroscopic approach using the Carto mapping system for ablation of the cavotricuspid isthmus in the United States. The approach is feasible and effective in achieving bidirectional block with less average total procedure time.

\section{Determining the Incidence and Factors of Cardiotoxicity in Breast Cancer Patients Treated With Anthracycline and/or Trastuzumab-Containing Regimen at Aurora Health Care}

Stephanie Ghojallu, Matthew Rappelt, Han-Yang Chen, Ruth Perez, Lydia Garlie, Geoffrey Riddell, Yingying Gu, Jun Zhang, Rubina Qamar, Bijoy K. Khandheria, Arshad Jahangir, Yang Shi

Aurora Nursing Administration; Aurora Research Institute; University of Wisconsin-Milwaukee; Hematology/ Oncology, Aurora Health Care; Aurora Cardiovascular Services and Sheikh Khalifa bin Hamad Al Thani Center for Integrative Research on Cardiovascular Aging, Aurora Health Care

Background: Anthracycline and trastuzumab are common breast cancer treatments. While improving survival, they elevate risk of congestive heart failure. The incidence of cardiotoxicity (CTx) with these therapies varies in the literature from $10 \%$ to $59 \%$, higher than those reported in clinical trials $(4 \%-$ $10 \%)$ that excluded patients with preexisting cardiovascular comorbidities. Studies have failed to establish consensus on the risk factors for CTx associated with these therapies.

Purpose: We aim to determine the incidence and risk factors of CTx in breast cancer patients treated with anthracycline and/or trastuzumab at Aurora Health Care.

Methods: A retrospective review of patients with breast cancer who received anthracycline and/or trastuzumab from 2002 to 2011 yielded a total of 2,383 patients. Patients with a left ventricular ejection fraction (LVEF) recorded prior to treatment and at least one follow-up LVEF were included in analysis ( $n=319,13.4 \%$ of total cohort). Database queries and electronic medical records review (assisted by an inhouse natural language processing tool) retrieved data on demographics, comorbidities, congestive heart failure symptoms, oncological treatments and LVEF. The study outcome was CTx, defined as a $\geq 10 \%$ decrease in LVEF to a level of $<55 \%$. Chi-squared and Fisher's exact tests were used for categorical variables to test differences in patient characteristics by CTx status (yes/no). Multivariate logistic regression analyses examined the association between risk factors and CTx.

Results: Average age of the patients was $54.9 \pm 12.1$ years; the cohort was comprised of $50.5 \%$ with obesity, $44.2 \%$ with smoking history and $47.3 \%$ with hypertension. A total of 79 patients developed CTx, an incidence of $24.8 \%$. Multivariable analysis identified divorced/widowed marital status (odds ratio [OR]: 2.70, 95\% confidence interval [CI]: 1.26-5.77), history of structural/electrophysiological (EP) cardiac disease (OR: 2.66, 95\% CI: 1.24-5.70) and combined anthracycline-trastuzumab therapy (OR: 2.92, 95\% CI: 1.48-5.77) as significant risk factors for CTx.

Conclusion: The incidence of CTx was greater in a community setting for which cardiac history and comorbidities are more diverse than in clinical trials. Consistent with prior literature, our study identified combined treatment with anthracycline and trastuzumab as a risk factor for CTx. Our study also suggests divorced/widowed marital status and prior structural/EP cardiac disease as additional risk factors for CTx. Further prospective studies are warranted for verification. We advocate for pre- and posttreatment cardiac monitoring of patients receiving these two therapies.

\section{Are There Advantages to Hiring In-House Training Program Graduates?}

Jessica J.F. Kram, Dennis J. Baumgardner

Department of Family Medicine, Aurora UW Medical Group; Center for Urban Population Health

Background: Several studies have compared international graduates on measures of performance, quality and satisfaction. No studies have compared internally versus externally hired graduates in relation to these measures.

Purpose: To identify if there is a difference in hiring patterns and care management (CM)/patient satisfaction (PS) scores between internal and external graduate hires.

Methods: We conducted a quality improvement study on graduates hired by Aurora Health Care from Jan. 1, 2006, to Dec. 14, 2015. CM scores were determined based on hire date. PS scores were calculated based on the calendar year, regardless of exact hire date. PS scales for scoring changed in mid-2010. Hired graduates with no CM and PS scores, as well as those with less than one year of employment, were excluded. Means were compared using two-sample t-tests and regression analysis. Categorical variables were analyzed using chi-squared and Fisher's exact test, as appropriate.

Results: Study population $(\mathrm{N}=108)$ characteristics included: mean age 38.0 years, $62.0 \%$ female gender and $56.5 \%$ 